\title{
How to Decompose a Binary Matrix into Three $h v$-convex Polyominoes
}

\author{
Andrea Frosini and Christophe Picouleau \\ Dipartimento di Sistemi e Informatica, Università di Firenze, \\ Viale Morgagni, 65 - 50134 Firenze - Italy \\ Laboratoire CEDRIC, CNAM \\ 292 rue St Martin, 75003 Paris - France \\ andrea.frosini@unifi.it, christophe.picouleau@cnam.fr
}

\begin{abstract}
Given a binary matrix, deciding wether it can be decomposed into three $h v$-convex matrices is an $\mathcal{N} \mathcal{P}$-complete problem, whereas its decomposition into two $h v$-convex matrices or two $h v$-polyominoes can be performed in polynomial time. In this paper we give a polynomial time algorithm that decomposes a binary matrix into three $h v$-polyominoes, if such a decomposition exists. These problems are motivated by the Intensity Modulated Radiation Therapy (IMRT).
\end{abstract}

Keywords: computational complexity, matrix decomposition, convex polyomino.

\section{Introduction}

The problem of the minimum decomposition of an integer matrix finds its practical applications in the Intensity Modulated Radiation Therapy (IMRT). For cancer treatment IMRT consists in delivering a radiation dose to destroy the tumor while maintaining the functionality of organs surrounding the tumor. The collimator is the medical machine that delivers the radiation dose (dose matrix). Technically, the collimator cannot deliver all shapes of matrices. The shape decomposition problem consists in decomposing the dose matrix into a set of deliverable shape matrices. In the literature, the decomposition into consecutive ones matrices is the most studied [15612. Baatar et al. 1] show that the problem of deciding wheter a binary matrix $A$ can be decomposed into at most $K h$-convex matrices is $\mathcal{N} \mathcal{P}$-complete in the strong sense even in the case where $A$ has one single row.

Motivated by a theoretical point of view, Jarray et al. [17] consider the problem of deciding wheter it is possible to decompose a binary matrix into a (small) fixed number of binary matrices fulfilling some specific requirements as it is usual in the field of the theoretical discrete tomography. They show that the decomposition into three $h v$-convex matrices is $\mathcal{N} \mathcal{P}$-complete in the strong sense whereas the decomposition problem into two $h v$-convex matrices is polynomial even in the case where each of the two matrices is an $h v$-polyomino. Note that this last requirement is of great importance for the applications in IMRT.

R. Gonzalez-Diaz, M.-J. Jimenez, B. Medrano (Eds.): DGCI 2013, LNCS 7749, pp. 311-322, 2013.

(C) Springer-Verlag Berlin Heidelberg 2013 
Following the work of Jarray et al. 17] we study the decomposition of a binary matrix into three $h v$-polyominoes. We design a polynomial time algorithm that given a binary matrix that either returns a decomposition into three $h v$ polyominoes or failure if such a triplet of $h v$-polyominoes does not exist.

The paper is organized as follows: in the following section we give the definitions, and the basic notions about convex polyominoes. In Section 3 we give the polynomial time algorithm that decomposes a binary matrix into three $h v$ polyominoes if such a decomposition exists. In the last section we conclude and some directions for future works are given.

\section{Definitions and Notations}

Let us consider binary $m \times n$ matrices and let us graphically represent them by sets of cells on a squared surface in a standard way, i.e. the 1-elements and 0-elements correspond to the presence or the absence of a cell in the related position. A binary matrix is horizontally convex, briefly $h$-convex, if the 1-elements of each row are connected; similarly, we say that it is vertically convex, briefly $v$-convex, if the 1-elements of each column are consecutive. Finally, an $h v$-matrix is a matrix that is horizontally and vertically convex (see Fig. 11).
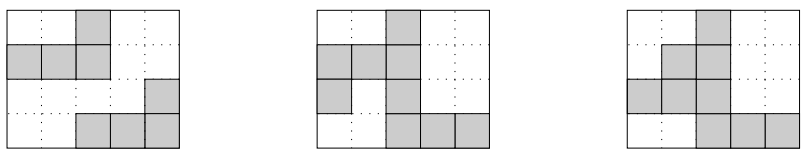

Fig. 1. From the left to the right: a $h$-convex matrix, a $v$-convex polyomino, a $h v$-convex polyomino

A binary matrix is a polyomino, if its elements are connected w.r.t. the side adjacency relation. In the sequel, we consider matrices that are both $h v$-convex and polyominoes, i.e. $h v$-polyominoes. Polyominoes, first introduced in [14], are well known combinatorial objects, and some of their subclasses are widely studied both in Combinatorics, and in Discrete Tomography. In the first area, there are many enumeration results according to different parameters 47810, that lead, among others, to efficient exhaustive and random generation algorithms 1116, while in the second area, their geometrical aspects are studied by means of quantitative data, called projections, about the number of cells that lie on parallel lines having discrete directions 2.3] or that lie inside small areas of fixed shape [13] (for an overview of the topic see [15]).

We consider the rows and the columns of a $m \times n$ matrix numbered in the standard way, i.e. downward from row 1 to row $m$, and rightward from column 1 ro column $n$. These few notions are enough to state the problem 3-decomposition we are going to study:

given a binary matrix A, we want to efficiently decompose it into at most three hv-polyominoes, if possible, otherwise give failure. 
To accomplish this task we will define a polynomial time (w.r.t. the dimension of $A$ ) algorithm 3-phv-DEC that returns either at most three $h v$-polyominoes $X$, $Y$, and $Z$ such that $A=X+Y+Z$ or failure when such a decomposition does not exist. Note that a strategy that fulfills this task gives also an answer in polynomial time to the related decision problem.

Our strategy will use the result of Proposition 1, that directly follows from an alternative definition of $h v$-polyomino: for any two elements $(i, j)$ and $\left(i^{\prime}, j^{\prime}\right)$ of a polyomino $A$, a path from $(i, j)$ to $\left(i^{\prime}, j^{\prime}\right)$ is a sequence $\left(i_{1}, j_{1}\right), \ldots,\left(i_{r}, j_{r}\right)$ of distinct adjacent elements of $A$, with $(i, j)=\left(i_{1}, j_{1}\right)$ and $\left(i^{\prime}, j^{\prime}\right)=\left(i_{r}, j_{r}\right)$. For each $1 \leq k \leq r-1$, we say that the two consecutive cells $\left(i_{k}, j_{k}\right),\left(i_{k+1}, j_{k+1}\right)$ form

- an east step if $i_{k+1}=i_{k}$ and $j_{k+1}=j_{k}+1$;

- a north step if $i_{k+1}=i_{k}-1$ and $j_{k+1}=j_{k}$;

- a west step if $i_{k+1}=i_{k}$ and $j_{k+1}=j_{k}-1$;

- a south step if $i_{k+1}=i_{k}+1$ and $j_{k+1}=j_{k}$.

We define a path to be monotone if it is entirely made of only two of the four types of steps defined above. So, in [9], the following definition is given: a polyomino is $h v$-convex if and only if every pair of cells is connected by a monotone path.

Proposition 1. Let $(i, j)$ and $\left(i^{\prime}, j^{\prime}\right)$ be two elements of the border of an $h v$ polyomino $A$. If we replace the path $\pi$ that connects them and runs along the border of $A$, with any another monotone path $\pi^{\prime}$ that connects $(i, j)$ to $\left(i^{\prime}, j^{\prime}\right)$, then the hv-convexity is preserved in the new polyomino.

The intuitive idea of the replacement of the border path $\pi$ with another monotone border path $\pi^{\prime}$ in $A$ having the same starting and ending positions, is that of inserting a 1-element in each position of $\pi^{\prime}$, then set to 0 the elements in the exterior of $\pi^{\prime}$, w.r.t. $A$, and to 1 those inside $A$, the whole process has to be carried on without compromising the connectedness of $A$.

We define a monotone path connecting the cells $a$ and $b$ to be maximum [resp. minimum] if each of its elements has the maximum [resp. minimum] row index w.r.t. the element in the correspondent positions in all the other monotone pathes that lead from $a$ to $b$ (note that, by definition, all the monotone pathes that lead from $a$ to $b$ have the same number of elements).

\section{A Fast Algorithm to Solve 3-decomposition}

The first step in defining a valid strategy to solve 3-decomposition for a generic binary matrix $A$, consists in cutting off some trivial cases that allow us to immediately identify a solution or state that no solution exists.

So, we start by giving $A$ as input to the algorithm 2-phv-DEC defined in [17, and that solves 2-decomposition. So, if 2-phv-DEC decomposes $A$, then a solution to 3-decomposition has been reached as well, otherwise, if it fails, we start the search for a decomposition into exactly three $h v$-polyominoes, if possible. It is straightforward that if the matrix $A$ is composed by at least two non connected 
components, then 3-decomposition can be solved in polynomial time by means of a run of 2-phv-DEC on each component.

As a consequence, the strategy to solve 3-decomposition we are going to define, considers only input matrices $A$ having one single connected component, i.e. polyominoes. It consists in the labeling of each cell of $A$ with one label $l \in\{x, y, z\}$, according to the belonging to one of the three $h v$-polyominoes, indicated by $X, Y$ and $Z$, we are trying to split $A$ into.

\subsection{Starting the Labeling Process}

We continue indicating with $A$ the input polyomino of 3-decomposition. First, we observe that the convexity of the polyominoes $X, Y$ and $Z$ implies that if two elements of $A$ with the same label lie on the same row [resp. column], then all the elements between them must belong to $A$, and share the same label. Second if two elements belong to two disconnected components of a row [resp. column], then they have different labels. During the labeling process we often use this property in order to assign labels by convexity.

We define a row of $A$ to be a $k$-row if it is constituted by exactly $k+1$ non connected sequences of elements; the cells between two consecutive of these sequences form a hole; these same definitions can be given for columns (in Fig. 2 the two disconnected components of the extremal rows form two holes). By convexity, it is immediate to realize that if $A$ has a $k$-row [resp. $k$-column] with $k \geq 3$, then 3 -decomposition has no solution.

Proposition 2. If 3 -decomposition has a solution for $A$, then each 2-row of A has a labeling of the form

$$
0^{k_{1}}\left(L_{1}\right)^{k_{2}} 0^{k_{3}}\left(L_{2}\right)^{k_{4}} 0^{k_{5}}\left(L_{3}\right)^{k_{6}} 0^{k_{7}}
$$

with $k_{2}, \ldots k_{6}$ different from zero, $k_{1}+\cdots+k_{7}=n$, and $L_{1}, L_{2}$, and $L_{3}$ being the three different labels.

We say that in a labeling like that of Proposition 2 the labels alternate as $L_{1} L_{2} L_{3}$. It is noteworthy that the connectedness of the three polyominoes $X, Y$ and $Z$ imposes that all the 2-rows [resp. 2-columns] of $A$ have the same alternation of labels, see Fig. 2. This property can be extended by connectedness to two generic rows of $A$ even if not the three labels are present, i.e. admitting one or more among the $k_{i}$ s, with $i=2,4,6$, to be zero:

Proposition 3. Let $i_{1}$ and $i_{2}$ be two rows of $A, i_{1}$ being a 2-row. If 3 -decomposition has a solution for $A$, then $i_{1}$ and $i_{2}$ have the same alternation of the labels.

This last proposition plays a special role in our decomposition strategy, since it allows to unambiguously assign an alternation of labels to the matrix $A$. Note that if no 2-rows are present in the matrix, then different alternations of variables can be possible, however, we underline that their number is six at most.

Finally, a similar definition of label alternation can be stated for the columns of $A$. So, from now on, we assume w.l.g. that $A$ has the row alternation $x y z$. 
Afterwards we fix one column alternation among the six permutations of $\{x, y, z\}$ (we will require 3-phv-DEC to carry on the six combinations).

The labeling process we are going to define consists of two main steps, the first one takes care of the areas of $A$ that lie between two 2-rows, and the second one that considers the remaining part of $A$, i.e. those areas that are delimited by at most one single 2-row.

\section{Labeling the Area between Two 2-Rows}

Consider the area $2 A$ between two 2-rows $i_{1}$ and $i_{2}$, with $i_{1}<i_{2}$, and let the leftmost and rightmost cells of the sequence of labels $x$ in row $i_{1}$ [resp. $i_{2}$ ] be $x_{l}^{1}$ and $x_{r}^{1}$ [resp. $x_{l}^{2}$ and $x_{r}^{2}$ ]; identify in a similar way the extremal cells of the sequences of labels $y$ and $z$, as shown in Fig. 2 .

Since the border of $A$ connects, on the left, the cells $x_{l}^{1}$ and $x_{l}^{2}$ of $2 A$, and, on the right, the cells $z_{l}^{1}$ and $z_{l}^{2}$ of $2 A$, it holds:

Theorem 1. If 3-decomposition has a solution for $A$, then the elements of the border of $A$ inside $2 A$ that connects the cells $x_{l}^{1}$ and $x_{l}^{2}$ [resp. $z_{l}^{1}$ and $z_{l}^{2}$ ] have label the $x$ (see Fig. Q (a)) [resp. z].

We assume that no 1-rows lie inside $2 A$, and we label the cells of this area according to the mutual positions of the extremal labeled cells of the 2-rows:

Proposition 4. If 3-decomposition has a solution for $A$, and the area $2 A$ has no 1-rows, then there also exists an (eventually different) decomposition such that the cells of $2 A$ till the border between $X$ and $Y$ are labeled as follows, according to the mutual positions of the extremal cells of the sequences $x$ and $y$ of the 2rows: let us assume that the index of the column of $y_{l}^{1}$ is greater than or equal to that of $y_{l}^{2}$, briefly $y_{l}^{1} \geq y_{l}^{2}$

Case $i: x_{r}^{1} \geq y_{l}^{2}$. Each cell $c \in 2 A$ not already labeled such that

1. $c<\overline{y_{l}^{2}}$, has label $x$;

2. it belongs to the minimum monotone path leading from $y_{l}^{2}$ to $y_{l}^{1}$, has label $y$, as shown in Fig. 2, (a);

Case ii: $x_{r}^{2}<x_{r}^{1}<y_{l}^{2}$. Each cell $c \in 2 A$ not already labeled such that

1. $c \leq x_{r}^{1}$, has label $x$;

2. $x_{r}^{1}<c<y_{l}^{2}$, has the label $x$ or $y$ according to the column alternation of A;

3. it belongs to the minimum monotone path leading from $y_{l}^{2}$ to $y_{l}^{1}$, it has label $y$;

Case iii: $x_{r}^{1}<x_{r}^{2}$. Each cell $c \in 2 A$ not already labeled such that

1. $c \leq x_{r}^{2}$, has label $x$;

2. $x_{r}^{2}<c<y_{l}^{2}$, has the label $x$ or $y$ according to the column alternation of A;

3. it belongs to the minimum monotone path leading from $y_{l}^{2}$ to $y_{l}^{1}$, it has label $y$.

Proof. Case $i$ is a direct consequence of Proposition 1, as shown in Fig. 2, (a). Case $i i$ : the labelings defined in 1 , and 3. resemble those of Case $i$, while 2 . is performed according to the column alternation of the labels, say $L_{1} L_{2} L_{3}$. The following cases arise: 
$a$. if at least one of the columns from the cell $x_{r}^{1}$ to the cell $y_{l}^{2}$ is a 2-column, then $c$ has label $L_{2}$;

$b$. if the column immediately on the right of $x_{r}^{1}$ has no cells above the area $2 A$, then $c$ has label $x$;

$c$. if the column immediately on the right of $x_{r}^{1}$ has no cells below the area $2 A$, then $c$ has label $y$.

In all these three cases, we are not able to set a labeling that maintains, at the same time, the monotonicity of the right border of $X$ and of the left border of $Y$, as in Case $i$. So, we determine the label of at least one cell inside the area defined in 2., and we extend it to all the remaining cells, obtaining the monotonicity of one of the two borders, and consequently preserving the convexity of both the polyominoes $X$ and $Y$. In case $a$., some cells inside the area of 2 . already have the label $L_{2}$ by the column alternation of the labels, in case $b$. (Fig. 2, (b)) the cells in the columns rightward $x_{r}^{2}$, and in row $i_{2}+1$ can not have label $x$, so the cells inside the area 2 . can have label $x$ without compromising the convexity of the polyomino $X$ in the solution, and leaving the path of the right border of $Y$ monotone and minimum in $2 A$. Finally, in case $c$. the cells in the columns leftward $y_{l}^{1}$, and in row $i_{1}-1$ can not have label $y$, so the cells inside the area 2. can have label $y$ without compromising the convexity of the polyomino $Y$ in the solution, and leaving the path of the left border of $X$ monotone in $2 \mathrm{~A}$.

Case iii can be treated as Case $i$.

Note that the labeling of the right border of $X$, and consequently of the left border of $Y$, when the extremal cells $x_{r}^{1}, y_{l}^{1}, x_{r}^{2}$, and $y_{l}^{2}$ of the 2-rows have the remaining three different mutual positions can be obtained by symmetry. Still by symmetry, we also obtain the labeling of the right border of $Y$, and consequently of the left border of $Z$, giving a complete labeling of the $2 A$ area when no 1-rows are present inside.

The labeling defined in Proposition 4 will be useful in the whole decomposition process, since it can be used each time two holes labeled as in Fig. 3, (a) (or, by extension, having the same labeling) have to be connected with a minimum monotone path.

Now we approach the problem of labeling the same area $2 A$ when 1-rows are present inside, by showing how to set the labels at the border of each of their holes, if not already provided, in order to connect them using Proposition 4 the row alternation is $x y z$, and the column alternation is fixed as well.

Proposition 5. Let $i$ be a 1-row of $A$ lying in the $2 A$ area between the 2 -rows $i_{1}$ and $i_{2}$, that contains a hole from the column $j$ to the column $j^{\prime}$. If a solution to 3-decomposition for $A$ exists, then there exists a solution such that the labeling of the elements of the border of the hole resembles one of those depicted in Fig 3 , according to the alternation of the labels in the columns.

The proof is a direct consequence of the convexity both of the rows and of the columns surrounding the hole.

We remark that each hole lies on the border between $X$ and $Y$ or $Y$ and $Z$, but such a choice is not always deterministic, i.e. they can lie on different 


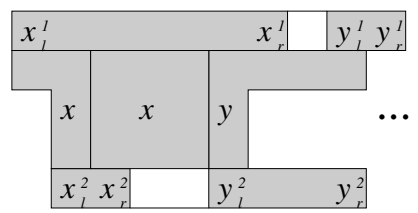

(a)

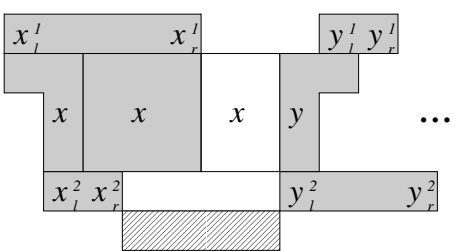

(b)

Fig. 2. Two examples of labelings of the area $2 A$ for cases $i,(a)$, and $i i,(b)$. In $(a)$ the labeling of the areas are set, from left to right, as follows: first by the labeling of the border of $A$, then by subcases 1 , and 2 . In $(b)$ the labels are set, from left to right, as follows: the first area by the labeling of the border of $A$, then by subcases 1,2 , and 3 . Note that, for subcase 2 , is represented the situation $b$, where the cells above the hole in row $i_{1}$ do not belong to $A$, and the dashed area below the hole of row $i_{2}$ can not have any cell with label $x$.

\begin{tabular}{|l|l|l|l|l|l|l|}
\hline & $x_{i}$ & $x$ & $x$ & $x$ & & \\
\hline & $x_{r}$ & & & & $y_{l}^{i}$ & \\
\hline & & $y$ & $y$ & $y$ & $y$ & \\
\hline
\end{tabular}

(a)

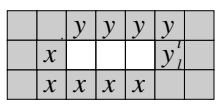

(b)

\begin{tabular}{|l|l|l|l|l|l|l|}
\hline & $y$ & $y$ & $y$ & $y$ & & \\
\hline$y$ & & & & $z$ & \\
\hline & & $z$ & $z$ & $z$ & $z$ & \\
\hline
\end{tabular}

(c)

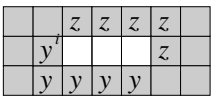

(d)

Fig. 3. The labeling of the hole in $(a)$ requires that the label $x$ precedes the label $y$ in the column alternation, while the labeling in $(b)$ requires that the label $y$ precedes the label $x$. Similarly it holds in $(c)$ and $(d)$ with the labels $y$ and $z$. The four cases admit the row alternation $x y z$.

borders, for different solutions of 3-decomposition. However, if some cells around it have already been labeled, then Fig. 3 shows how to extend these labels to the whole border of the hole. To carry on this labeling, we consider the mutual positions of all the holes inside $2 A$ and of the holes in the rows $i_{1}$ and $i_{2}$.

Assuming that the column alternation of labels to be $x y z$, we arrange the holes of the 1 -rows inside $2 A$ in a sequence $\sigma=\left\{\left(i_{1}^{\prime}, j_{1}^{\prime}\right), \ldots,\left(i_{k}^{\prime}, j_{k}^{\prime}\right)\right\}$, according to the column indexes of their leftmost (void) cells, and if two cells lie in the same column, then they are arranged in decreasing order w.r.t. their row indexes. Note that the internal holes whose column indexes intersect those of the holes in the row $i_{1}$ or $i_{2}$ have already been labeled, since they are 2-columns, and the same holds for the holes that share at least one column. We recall that an element $(i, j)$ of $\sigma$ is a left-to-right minimum if for each element $\left(i^{\prime}, j^{\prime}\right)$ that lies on its left in $\sigma$, it holds $i \leq i^{\prime}$; the definition of right-to-left maximum is similar.

Proposition 6. If a solution of 3-decomposition for $A$ exists, then the non already labeled hole $\left(i_{t}^{\prime}, j_{t}^{\prime}\right)$, with $1 \leq t \leq k$, has label as in Fig. 3, (a) if it is a left-to-right minimum, and not a right-to-left maximum (w.r.t. the row indexes) for the sequence $\sigma$. 
The proof follows from the convexity of the polyominoes $X, Y$, and $Z$. We observe that, if in the column alternation of $A$, the label $y$ precedes the label $x$, then the following changes has to be done: the sequence $\sigma$ is defined on the rightmost cell of each hole, and in Proposition 6, the hole $\left(i_{t}^{\prime}, j_{t}^{\prime}\right)$ is a right-to-left minimum and its labeling is as in Fig. 3. (b). A similar labeling can be defined for the holes that lie on the border between $Y$ and $Z$. As a neat consequence of the proof of Proposition 6, it holds:

Corollary 1. If there exists an element of $\sigma$ that is neither a left-to-right minimum nor a right-to-left maximum, then 3-decomposition has no solution.

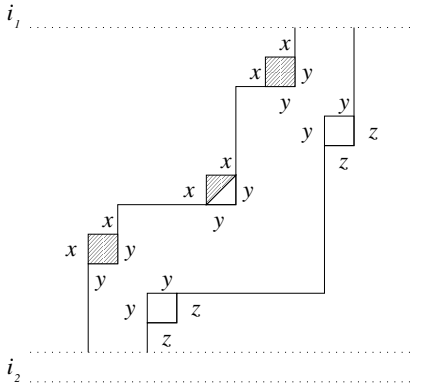

(a)

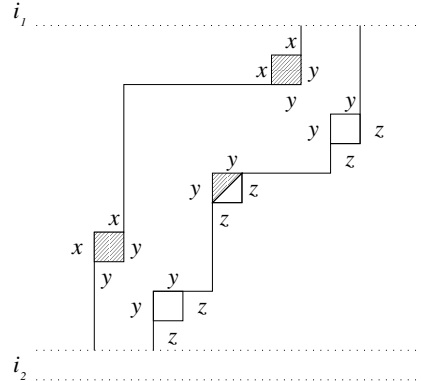

(b)

Fig. 4. In $(a)$, and (b) the two possible labelings of the holes internal to a $2 A$ area, when a hole that is both left-to-right minimum (dashed holes) and right-to-left maximum (blank holes) is present. The border between $X$ and $Y$ is the minimum one, while the one between $Y$ and $Z$ is the maximum.

Now we consider the case of a non labeled hole that is both left-to-right minimum and right-to-left maximum: we observe that it may allow two different labelings, i.e., with the assumed column alternation of labels, those of Fig. $3(a)$ and $(c)$, as shown in Fig. 4, by the half dashed hole.

However, if a hole is a left-to-right minimum and a right-to-left maximum, then there exists a hole on its right or on its left that is a left-to-right minimum or a right-to-left maximum, respectively. The label of the hole is the following:

Proposition 7. Let the cell $\left(i_{t}^{\prime}, j_{t}^{\prime}\right) \in \sigma$ be both a left-to-right minimum and a right-to-left maximum. If there exists a cell $\left(i_{s}^{\prime}, j_{s}^{\prime}\right) \in \sigma$ that is a left-to-right minimum and not a right-to-left maximum, with $j_{t}^{\prime}<j_{s}^{\prime}$, then there exists a solution of 3 -decomposition for $A$ where the hole in $\left(i_{t}^{\prime}, j_{t}^{\prime}\right)$ has its labeling as in Fig. $3(a)$. If the cell $\left(i_{s}^{\prime}, j_{s}^{\prime}\right)$ does not exist, then the hole in $\left(i_{t}^{\prime}, j_{t}^{\prime}\right)$ has its labeling as in Fig. $3(c)$.

The proof is immediate since the presence of the left-to-right minimum $\left(i_{s}^{\prime}, j_{s}^{\prime}\right)$ assures that the polyomino $X$ extends till the column $j_{t}^{\prime}$, since $j_{t}^{\prime}<j_{s}^{\prime}$. 
A similar reasoning holds in the case that there exists a cell $\left(i_{s}^{\prime}, j_{s}^{\prime}\right) \in \sigma$ that is a right-to-left maximum, and not a left-to-right minimum, with $j_{t}^{\prime}>j_{s}^{\prime}$.

This last observation completes the labeling process of the holes inside the $2 A$ area (when the label $x$ precedes the label $y$ in the column label alternation). So we can determine the border between $X$ and $Y$ by connecting, row after row from $i_{2}$ to $i_{1}$, the holes labeled with $x$ and $y$ using monotone minimum pathes.

\section{Labeling the Zone with Only One 2-Row or without 2-Rows}

Properties [6] and 7 give a valuable way of detecting the borders of $X, Y$, and $Z$ in presence of holes, and below it will be used in a more general setting.

So, let us assume that the column alternation of labels in $A$ is still $x y z$ and that, w.l.g., row $i$ is a 2-row, and, for each $i^{\prime}<i$, row $i^{\prime}$ is not; we indicate this area with $2 A^{-}$(in the sequel, $2 A^{-}$can also indicate an area where row $i$ is a 2-row, and, for each $i^{\prime}>i$, row $i^{\prime}$ is not, or the whole matrix if there are no 2-rows). Now, we proceed in labeling $2 A^{-}$: if no 1 -rows are present, then we act similarly to what performed in Proposition 4, considering as holes also those inside the 1-columns along the border of $A$, say border 1-columns. Those holes that do not belong to any 1-row, by the connectedness of $X, Y$, and $Z$, can lie only in this area, and their extremal cells must have different labels. Since we assumed the column alternation of labels to be $x y z$, then only three labelings are possible, i.e. those shown in Fig. 5. Consecutive border 1-columns whose holes share at least one row forms a border 1-columns block. The following is straightforward:

Proposition 8. In a $2 A^{-}$area, if the border 1-columns $j$ and $j^{\prime}$ belong to two different blocks, then the extremal cells of their holes can not have the same labeling.

By Property 8, it holds that the number of different labelings of the border 1columns in a $2 A^{-}$area is linear w.r.t. the number of columns, so we can consider each of them in a different line of computation, maintaining the polynomiality of the decomposition of $A$.
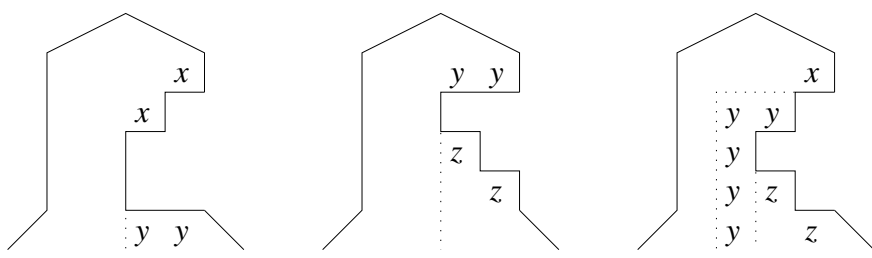

Fig. 5. The three possible labelings of blocks of border 1-columns in a $2 A^{-}$area, when the column alternation is $x y z$. The possible borders of $X, Y$ and $Z$ are set with dotted lines. Note that the rightmost example shows a situation where two different labelings of the holes are present in the same block. 
If there also exist some 1-rows in $2 A^{-}$, then we proceed in labeling them according to Properties [6] and [7. Now, all the holes inside $2 A^{-}$are labeled, and the decomposition procedure can proceed by detecting the border between $X$ and $Y$, and, using symmetry, the other one between $Y$ and $Z$.

Finally, if the polyomino $A$ contains neither 2-rows nor 2-columns, then the holes of its 1-rows, together with the holes of its border 1-columns, can be still labeled using the results of Properties 6 and 7

\section{The Decomposition Procedure}

Now, we are able to give the final procedure 3-phv-DEC that detects a solution to 3-decomposition on the polyomino $A$, if possible, by labeling its cells:

\section{Procedure: 3-phv-DEC}

Input: a binary matrix $A$.

Output: three $h v$-polyominoes $X, Y$, and $Z$ such that $A=X+Y+Z$, if possible, otherwise failure.

Step 1: run the procedure 2-phv-DEC defined in [17] on each connected component of $A$. If at most three $h v$-polyominoes are detected, then give them as output. Otherwise, if $A$ has one single connected component, then goto Step 2, else give failure;

Step 2: label the 2-rows of $A$ according to the row alternation $x y z$, and the 2-columns according to each of the six possible column alternation of labels. For each of them proceed to Step 3;

Step 3:

Step 3.1: for each $2 A$ area in $A$, label the border of $A$ with $x$ and $z$. If there are no 1-rows inside, then label the cells of $X$ as defined in Proposition 4. and symmetrically do the same with $Z$, else label the holes as in Properties [6] and 7. If there is a non labeled hole, then give failure;

Step 3.2: for each $2 A^{-}$area, label the holes of the 1-rows as in Properties 6] and [7, and for all possible combinations label the extremal cells of the holes of the border 1-columns according with the chosen column alternation. Note that this exhaustive labeling involves two different $2 A^{-}$ areas at most. Apply this labeling even in the case where $A$ does not contain any 2-row.

Step 4: for each $2 A$ area inside the 2-rows $i_{1}$ and $i_{2}$, consider the sequence $\sigma$ as defined in Proposition 6. Connect with the minimum monotone path the holes of the 1-rows whose border contains $x$ cells, acting as in Fig. 2, (a); the whole path will be part of the right border of $X$. Finally, connect the 2-row $i_{2}$ with the monotone path as described in Proposition 4, after considering as $x_{r}^{1}$ the cell $\left(i_{s}^{\prime}, j_{s}^{\prime}\right)$ that is the first element of $\sigma$ such that the cell $\left(i_{s}^{\prime}, j_{s}^{\prime}-1\right)$ has label $x$. The 2-row $i_{2}$ can be connected to the monotone path similarly. Act symmetrically to detect the border between $Y$ and $Z$;

Step 5: for each $2 A^{-}$area, act as in Step 4 connecting with the minimum monotone path the holes of the 1 -rows whose border contains $x$ cells, then connect the 2 -row of $2 A^{-}$with the monotone path. Complete the monotone path leading northward or southward, and maintaining its minimality (for 
the northward case, see Fig. 2, (b) for an example). Symmetrically, detect the border between $Y$ and $Z$. Finally, complete the labeling of the border of $A$ in the area;

Step 6: if the polyomino $A$ does not contain any 2-row, then connect the holes of the 1-rows as in Step 4 to find the border between $X$ and $Y$, and, symmetrically, the border between $Y$ and $Z$. Then complete the monotone path both northward and southward maintaining its minimality. Once found the internal border of the three polyominoes, complete the labeling of the cells at the border of $A$;

Step 7: complete the labeling of the internal cells of $A$ by convexity. If the three obtained polyominoes $X, Y$, and $Z$ are $h v$-convex, then give them as output, else give failure.

The correctness of the algorithm is assured by the fact that at each step we generate the border between $X$ and $Y$ by using minimum monotone pathes that connect all the detected $x$ cells. Since those cells either have to belong to $X$ for each solution of 3-decomposition (Properties 4 and [6), or can be connected to them by a monotone path (so they belong to at least one solution of 3decomposition, as stated in Proposition[7), then the correctness follows. The same reasoning holds for the border between $Y$ and $Z$. The computational complexity of the procedure is clearly polynomial, and it is performed for six times at most, one for each possible column alternation of the three labels. So the whole process turns out to be performed in polynomial time, as well.

\section{Conclusions and Further Works}

We designed a polynomial time algorithm that decomposes a binary matrix into three $h v$-polyominoes Recall that in contrast the decomposition into three $h v$-matrices is an $\mathcal{N} \mathcal{P}$-hard problem (see [17]). Now, a natural question is the following: given $k>3$, can this algorithm be adapted for the decomposition into $k h v$-polyominoes?

Other further works should be the following: motivated from a practical problem, give an extension of the algorithm in the three-dimensional case. Which kind of generalization should be the theoretical treatments in the higher dimensional discrete space.

\section{References}

1. Baatar, D., Hamacher, H.W., Ehrgott, M., Woeginger, G.J.: Decomposition of integer matrices and multileaf collimator sequencing. Discrete Applied Mathematics 152(1-3), 6-34 (2005)

2. Barcucci, E., Del Lungo, A., Nivat, M., Pinzani, R.: Reconstructing convex polyominoes from horizontal and vertical projections. Theoretical Computer Science 155, 321-347 (1996)

3. Barcucci, E., Frosini, A., Rinaldi, S.: An algorithm for the reconstruction of discrete sets from two projections in presence of absorption. Discrete Applied Mathematics 151(1-3), 21-35 (2005) 
4. Battaglino, D., Fedou, J.M., Frosini, A., Rinaldi, S.: Encoding Centered Polyominoes by Means of a Regular Language. In: Mauri, G., Leporati, A. (eds.) DLT 2011. LNCS, vol. 6795, pp. 464-465. Springer, Heidelberg (2011)

5. Boland, N., Hamacher, H., Lenzen, F.: Minimizing beam-on time in cancer radiation treatment using multileaf collimators. Networks 43(4), 226-240 (2003)

6. Bortfeld, T., Boyer, A., Kahler, D., Waldron, T.: X-ray field compensation with multileaf collimators. International Journal of Radiation Oncology, Biology, Physics 28(3), 723-730 (1994)

7. Bousquet-Mélou, M.: A method for the enumeration of various classes of columnconvex polygons. Discrete Mathematics 154, 1-25 (1996)

8. Castiglione, G., Frosini, A., Munarini, E., Restivo, A., Rinaldi, S.: Enumeration of L-convex polyominoes. II. Bijection and area. In: Proceedings of FPSAC 2005, \#49, pp. 531-541 (2005)

9. Castiglione, G., Restivo, A.: Reconstruction of $L$-convex Polyominoes. Electronic Notes in Discrete Mathematics 12, 290-301 (2003)

10. Delest, M., Viennot, X.: Algebraic languages and polyominoes enumeration. Theoretical Computer Science 34, 169-206 (1984)

11. Del Lungo, A., Duchi, E., Frosini, A., Rinaldi, S.: On the generation and enumeration of some classes of convex polyominoes. The Electronic Journal of Combinatorics 11, \#R60 (2004)

12. Ehrgott, M., Hamacher, H.W., Nußbaum, M.: Decomposition of Matrices and Static Multileaf Collimators: A Survey. In: Optimization in Medicine. Optimization and Its Applications, vol. 12, pp. 25-46 (2008)

13. Frosini, A., Nivat, M.: Binary Matrices under the Microscope: A Tomographical Problem. Theoretical Computer Science 370, 201-217 (2007)

14. Golomb, S.W.: Checker boards and polyominoes. American Mathematical Monthly 61(10), 675-682 (1954)

15. Herman, G.T., Kuba, A. (eds.): Discrete tomography: Foundations algorithms and applications. Birkhauser, Boston (1999)

16. Hochstätter, W., Loebl, M., Moll, C.: Generating convex polyominoes at random. In: Proceeding of the 5th FPSAC, Discrete Mathematics, vol. 153, pp. 165-176 (1996)

17. Jarray, F., Picouleau, C.: Minimum decomposition in convex binary matrices. Discrete Applied Mathematics 160, 1164-1175 (2012)

18. Shepard, D., Ferris, M., Olivera, G., Mackie, T.: Optimizing the delivery of radiation therapy to cancer patients. SIAM Review 41(4), 721-744 (1999) 